\title{
Research Paper \\ Prediction of Subscale Test Anxiety Considering Behavioral Procrastination, Decisional Procrastination and Cognitive Avoidance in University Students
}

\author{
*Yahya Hosseinzadeh Firouzabad', Soodabeh Bassak Nejad², Iran Davoudi ${ }^{3}$
}

1. MSc., Department of Clinical Psychology, Faculty of Education and Psychology, Shahid Chamran University of Ahvaz, Ahvaz, Iran.

2. PhD in Psychology, Associate Professor, Department of Clinical Psychology, Faculty of Education and Psychology, Shahid Chamran University of Ahvaz, Ahvaz, Iran

3. PhD in Psychology, Assistant Professor, Department of Clinical Psychology, Faculty of Education and Psychology, Shahid Chamran University of Ahvaz, Ahvaz, Iran.

\begin{tabular}{|l|l}
\hline $\begin{array}{c}\text { Use vourdevice toscan } \\
\text { and read the article online }\end{array}$ \\
Cltation: Hosseinzadeh Firouzabad Y, Bassak Nejad S, Davoudi I. [Prediction of Subscale Test Anxiety Considering \\
Behavioral Procrastination, Decisional Procrastination and Cognitive Avoidance in University Students (Persian)]. Iranian Journal \\
of Psychiatry and Clinical Psychology. 2018; 23(4):424-437. https://doi.org/10.29252/NIRP.IJPCP.23.4.424 \\
doI: https://doi.org/10.29252/NIRP.IJPCP.23.4.424
\end{tabular}

Received: 18 Sep. 2016 Accepted: 15 Apr. 2017

Key words: Behavioral procrastination, Decisional procrastination, Cognitive avoidance, Test anxiety

\section{ABSTRACT}

Objectives Test anxiety is the most important problem among students around the world. The purpose of this study was to investigate the relationship between behavioral procrastination, decisional procrastination and cognitive avoidance with subscales test anxiety in students of the Shahid Chamran University of Ahvaz.

Methods This survey study is correlation in form. A sample of 200 students was selected by multi- stage cluster sampling method. General procrastination scale, decisional procrastination scale, cognitive avoidance questionnaire and Fried-Ben test anxiety were used to collect data. In order to analyze data, canonical correlation and multiple regression methods were used. Data analysis was carried out using the SPSS-17 software.

Results The canonical analysis showed that the linear combinations of predictor variables were correlated with linear combinations of dependent variables. Regression analysis showed that procrastination behavioral and procrastination decisional significantly predicted social derogation $(\beta=0.238, P<0.001$; $\beta=0.302, P<0.001$, respectively) and cognitive obstruction $(\beta=0.381, P<0.001 ; \beta=0.204, P<0.006$, respectively). Also, procrastination behavioral and cognitive avoidance significantly predicted tenseness ( $\beta=0.314, P<0.001 ; \beta=0.246, P<0.002$, respectively).

Conclusion It is argued that universities should pay more attention to the factors that play significant roles in decreasing procrastination and decreasing cognitive avoidance. This is more likely to reduce test anxiety among graduate students.

\section{Extended Abstract}

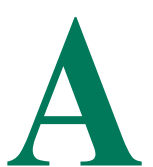

\section{Introduction}

nxiety, as a part of every person's life, is considered as an appropriate and consistent response. Lack of anxiety or excessive anxiety can lead to significant problems and dangers. In this research, the relationship between behavioral procrastina- tion, decisional procrastination, and cognitive avoidance with subscales test anxiety (Derogation, Obstruction, and Tenseness) have been investigated.

Empirical research has shown that anxiety is positively related to procrastination and plays a role in its formation. By eliminating the anxiety agent (evaluation position), the amount of procrastination in procrastinators is reduced [12]. Studies have showed that procrasti-

* Corresponding Author:

Yahya Hosseinzadeh Firouzabad, MSc.

Address: Department of Clinical Psychology, Faculty of Education and Psychology, Shahid Chamran University of Ahvaz, Ahvaz, Iran.

Tel: +98 (61) 33332618

E-mail: hosseinzadeh.yahya@gmail.com 
nation is associated with certain concerns such as fear of failure, test anxiety, social anxiety, and shyness [13]. Various research findings suggest that selecting one of the coping styles or combinations of them can help survive and face mental pressures. Meanwhile, avoidance coping is trying to avoid negative events, which can be manifested cognitively and behaviorally [19].

Most of the researchers, especially in the Iranian society, have not paid adequate attention to other aspects of procrastination (such as behavioral procrastination and decisional procrastination) and have considered cognitive avoidance with social anxiety. Therefore, by considering the effect of procrastination and cognitive avoidance in creating anxiety, this research attempts to determine whether behavioral procrastination, decisional procrastination, and cognitive avoidance are related to subscales test anxiety among students.

\section{Method}

The research method is a canonical correlation, which is the relationship between two variables, one of which is derived from the linear combination of independent variables and the other from the combination of dependent variables. The statistical population of this study included all male and female students studying in the academic year of 20152016 in the Shahid Chamran University of Ahvaz. In this research, multi-stage cluster sampling method was used to select 200 students for testing the hypotheses. In this way, five faculties were randomly selected; from each faculty, several classes were selected, and in each class, several students were selected to answer the questionnaires. For data collection, we used the General Procrastination scale, Decisional Procrastination scale, cognitive avoidance questionnaire, and Fried-Ben test anxiety questionnaire. The reliability of the scale was obtained using Cronbach's alpha.

Data analysis was carried out using the SPSS-17 software. In order to analyze the research hypotheses, parametric statistical methods were used. The canonical correlation analysis was used to study the prediction of a linear combination of criterion variables by a linear combination of predictor variables. The multivariate regressions analysis was conducted to study the multiple relationships between predictor variables with each of the criterion variables.

\section{Results}

The demographic information of the sample is shown in Table 1. The results of significant tests of canonical correlation analysis full model are presented in Table 2 . As seen in Table 2, Wilks's Lambda $(\mathrm{P}<0.001, \lambda=0.705, \mathrm{~F}=8.10)$ indi- cate that there is a significant relationship between the two categories variables (behavioral procrastination, decisional procrastination and cognitive avoidance with derogation, obstruction, and tenseness). Regarding the full model significance, the first hypothesis of the current study (prediction of subscale test anxiety considering predictor variables) is confirmed. Therefore, the model obtained in this study determines $29.5 \%$ of the variance between behavioral procrastination, decisional procrastination and cognitive avoidance (as a predictor variable) variables with derogation, obstruction, and tenseness (as a criterion variable). The results of multivariate regression analysis showed that behavioral procrastination and decisional procrastination significantly predicted derogation $(\beta=0.302, \mathrm{P}<0.001$ and $\beta=0.238, P<0.001)$ and obstruction $(\beta=0.381, P<0.001$ and $\beta=0.204, \mathrm{P}<0.006$ ) in students. Also, behavioral procrastination and cognitive avoidance predicted tenseness ( $\beta=0.314, \mathrm{P}<0.001$ and $\beta=0.246, \mathrm{P}<0.002$ ) in students.

\section{Discussion}

The results of the study showed that there is a significant relationship between the canonical predictor variables as behavioral procrastination, decisional procrastination and cognitive avoidance with the canonical criterion variables. In other words, these variables predict subscales test anxiety. Due to negligence, most of the students delay studying their lessons until the night of the exam. This results in anxiety before the exam [37], leading to academic failure. As a result, the students feel that they do not have the necessary qualifications, and their academic confidence is seriously damaged. According to Onwuegbuzie's hypothesis [38], anxiety and procrastination are in a two-way relationship and interact in a complicated way; hence, negligent students experience high levels of anxiety due to the feeling of threat to their self-esteem. High levels of anxiety postpone the probability of doing that task, to relieve the resulting suffering.

As stated previously, cognitive avoidance, as one of the predictor variables, can predict test anxiety. This result can be explained by the Lazarus and Folkman theories. These theorists believed that the person in the situation of stress or task first assesses his/her ability and the difficulty and threats of the task. If, based on this assessment, the situation is disturbing or unpleasant for a person, he or she experiences a negative emotion such as anxiety. Here, the person uses coping styles such as avoiding the situation to escape from anxiety. Therefore, the person who has test try to avoid situations and thoughts related to test that can also delay the doing of the task. 
Table 1. Mean, standard deviation, minimum, and maximum of variables

\begin{tabular}{ccccc}
\hline Variable & Mean & Standard Deviation & Minimum & Maximum \\
\hline Behavioral procrastination & 57.65 & 9.16 & 32 & 79 \\
Decisional procrastination & 15.16 & 3.90 & 5 & 118 \\
\hline Cognitive avoidance & 76.13 & 1.29 & 0 & 25 \\
Derogation & 11.04 & 5.32 & 0 & 21 \\
\hline Cognitive obstruction & 10.33 & 4.19 & 0 & 18 \\
Tenseness & 6.42 & 4.06 & 0 & 59 \\
\hline
\end{tabular}

Table 2. Results of significant tests of canonical correlation analysis

\begin{tabular}{cccccc}
\hline Test Name & Value & Approx. F & Hypoth. df & Error df & Sig. \\
\hline Pillais & 0.318 & 7.76 & 9 & 588 & 0.001 \\
Wilks & 0.705 & 8.10 & 9 & 472 & 0.001 \\
Hotellings & 0.384 & 8.23 & 9 & 578 & 0.001 \\
Roys & 0.208 & - & - & - & 0.001 \\
\hline
\end{tabular}

In summary, the results of the current study show that the people with behavioral procrastination, decisional procrastination, and cognitive avoidance experience more test anxiety and experience the fear of humiliation, irrational and negative thoughts, and more tenseness. In the end, it can be said that the lack of proper planning, lack of specific goals, and lack of proper problem-solving strategies, which refer to procrastination and avoidance characteristics, can increase anxiety. The limitations of this research include the mental state of the person at the time of completion of the questionnaire, time-consuming and honesty in responding, which is a basic condition of health and accuracy of research. Given that many students experience anxiety in a particular lesson, the anxiety of a particular lesson can be the subject of future research.

\section{Ethical Considerations}

Funding
This research was extracted from the MSc. thesis of the first author in the Department of Clinical Psychology, Faculty of Psychology and Educational Sciences, Shahid Chamran University of Ahwaz, Ahwaz, Iran.

\section{Conflict of Interest}

The authors declare no conflict of interest.

\section{Acknowledgements}

We are grateful to the professors and all the students participating in this research 


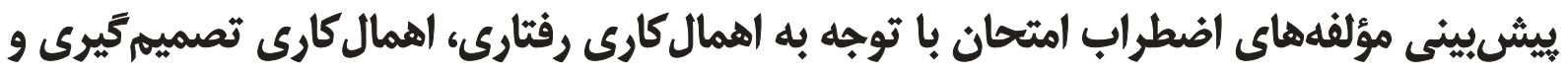 اجتناب شناختى در دانشجويان
}

\author{
"يحيى حسينزاده فيروزآباد'، سودابه بساكنزّاد'، ايران داودى"
}

\begin{abstract}
1- كارشناس ارشد، كروه روانشناسى بالينى، دانشكده روانشناسى و علوم تربيتى، دانشعاه شهيد جمران اهوازٔ، اهوازع، ايران.

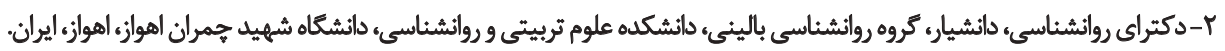

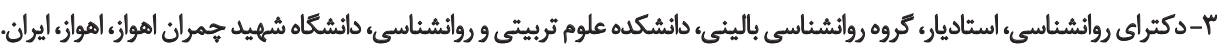

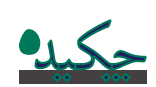

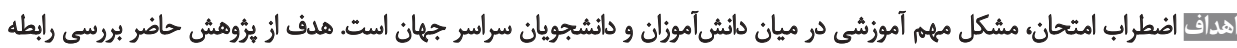

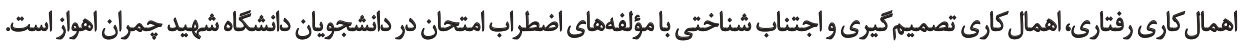

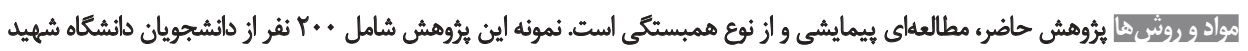

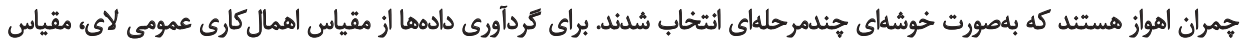

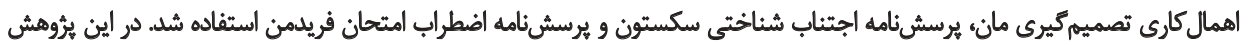

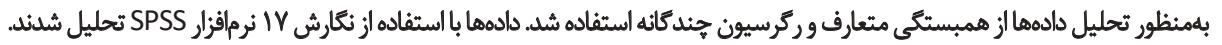

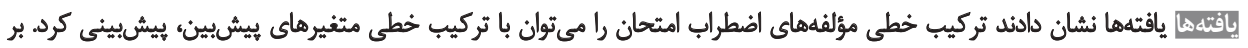

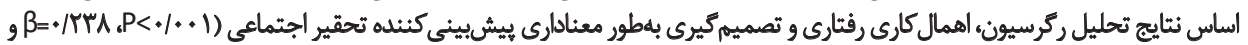

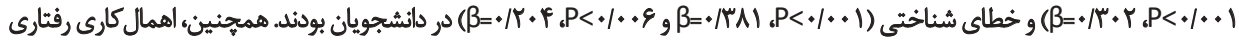

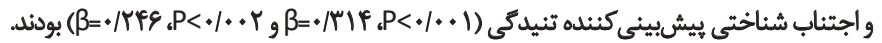

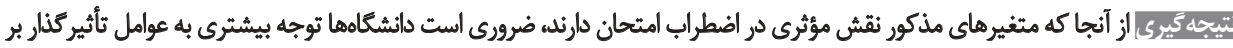

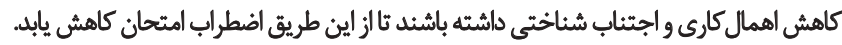

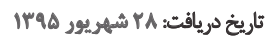
تاريخ بذيرش: و ثبروردين

براى بسيارى از دانشجويان ايجاد كثد [f]

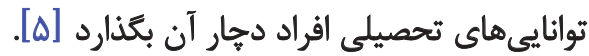

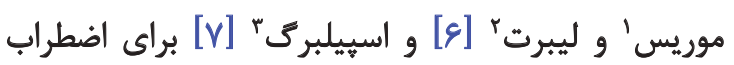

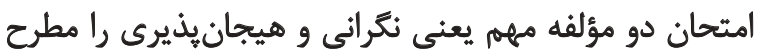

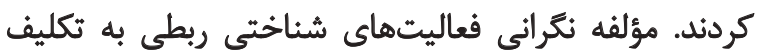

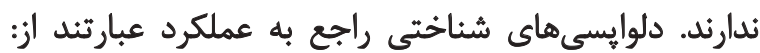

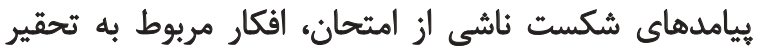

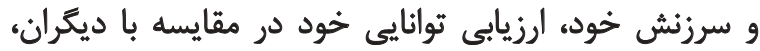

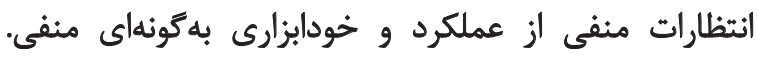

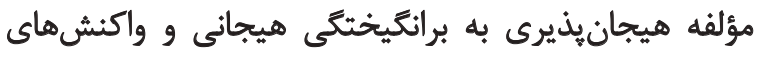

1. Morris

2. Liebert

3. Spilberger مقاd

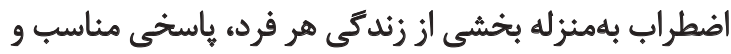

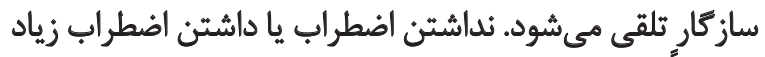

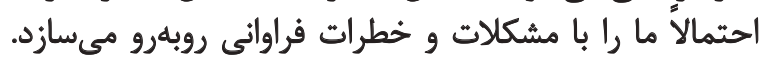

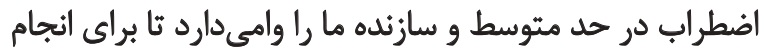

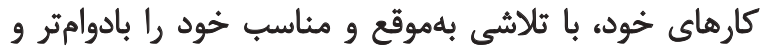

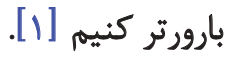

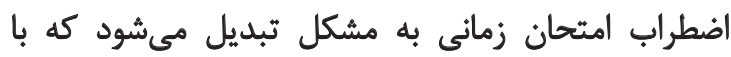

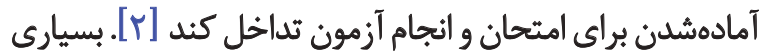

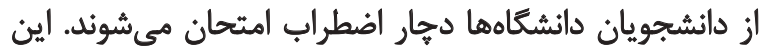

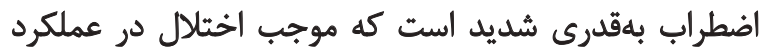

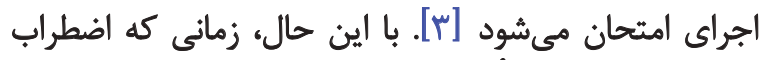

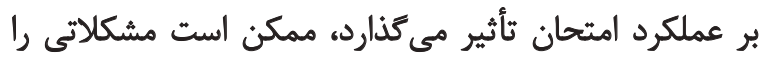




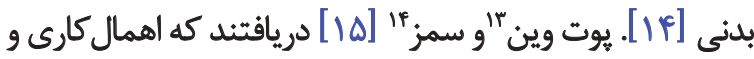

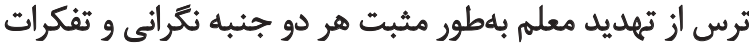
نامربوط به امتحان را ييشبينى ميلى مثند.

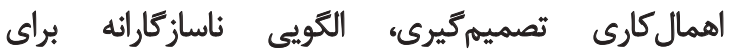

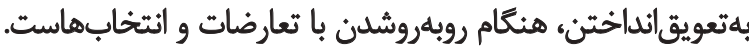

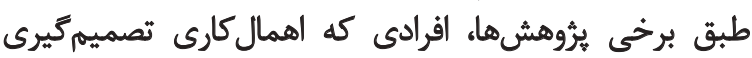

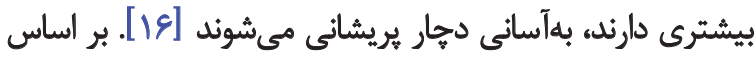

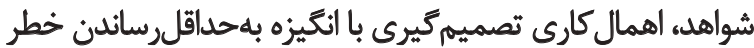

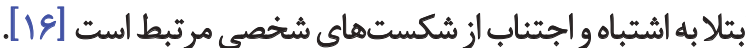

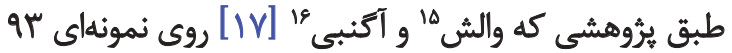
نفرى از دانشجويان در دانشكاه لندن انجام دادئ دادند، تعلل مانئد

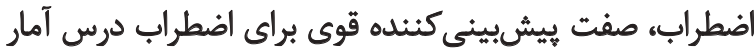

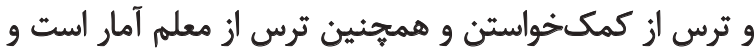

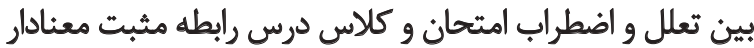

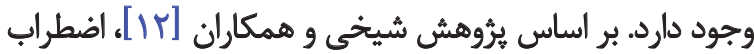

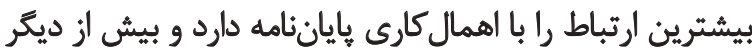

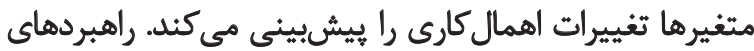

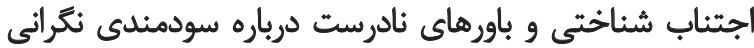

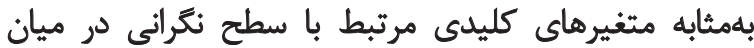

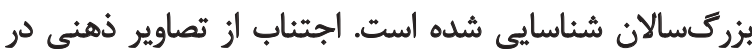

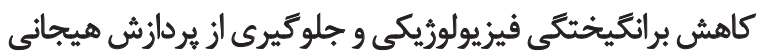

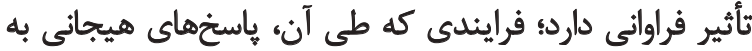

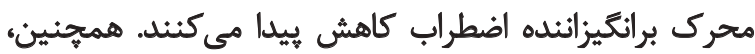

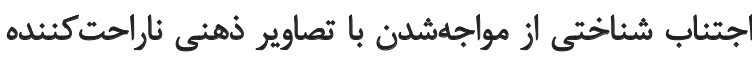

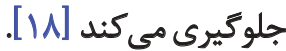

بر اساس طبقلبندى موريس و شافر ، سبكهاي مقابله مي توانئد

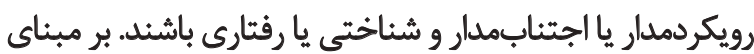

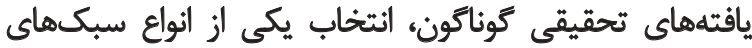

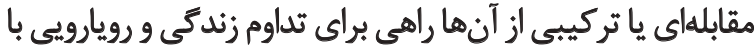

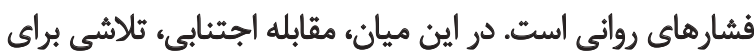

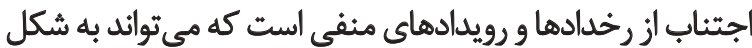

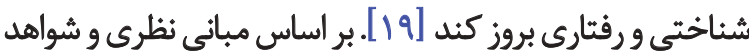

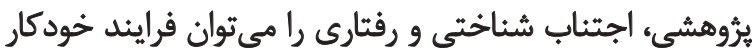

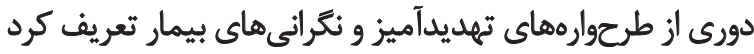

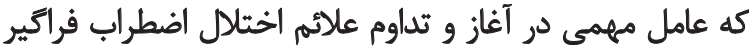

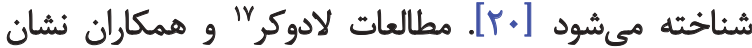

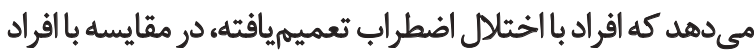

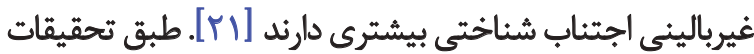

13. Putwin

14. Symes

15. Walsh

16. Agwunobi

17. Ladouceur

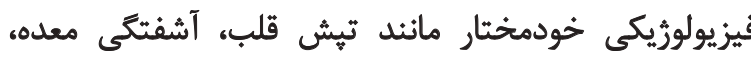
سردرد، تعريق، لززش و غيره اشاره دارد.

سازههاي مختلفى با اضطراب امتحان مرتبط هستند كه در

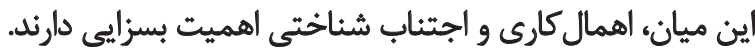

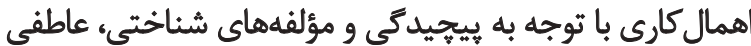

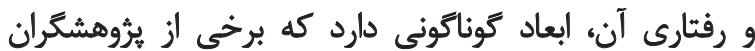

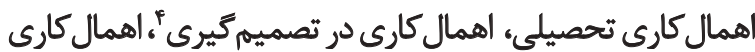

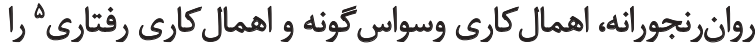

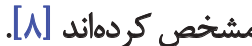

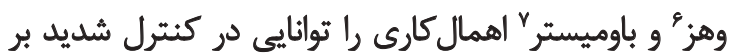

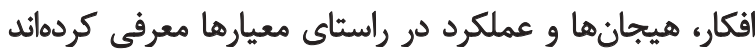

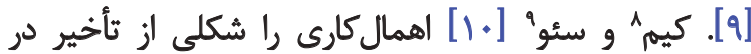

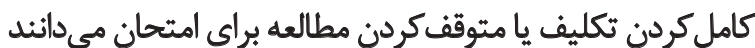

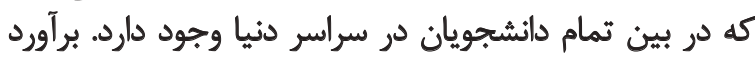

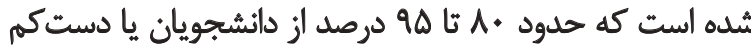

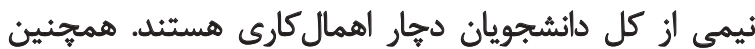

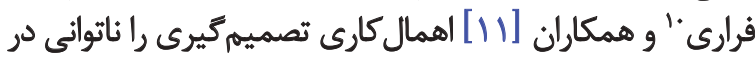

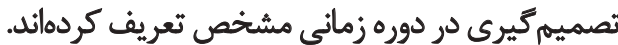

بر اساس تحقيقات شافر"و همكاران [F] درباره اضطراب

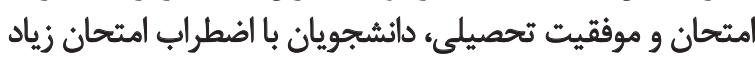

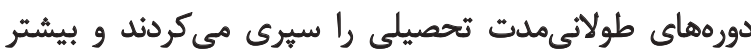

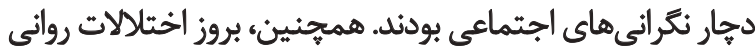

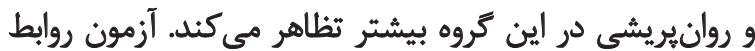

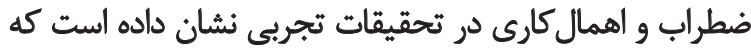

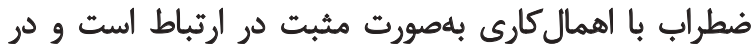

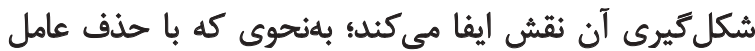

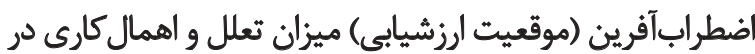

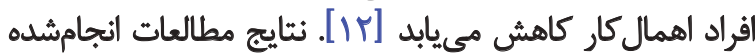

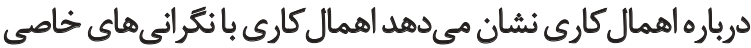

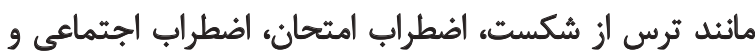

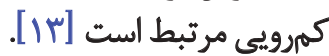

ساراسون"براى اضطراب امتحان جهار جنبه مشخص كرده

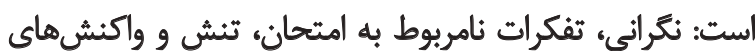

4. Decisional procrastination

5. Behavioral procrastination

6. Vohs

7. Baumeister

8. Kim

9. Seo

10. Ferrari

11. Schaefer

12. Sarason 
دانشجو بودن و برخوردارى نسبى از سلامت روان بود. همجئين

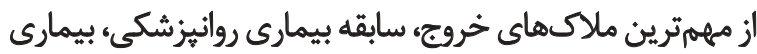

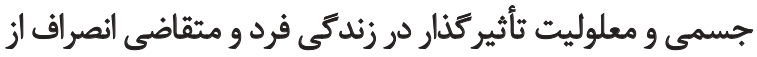

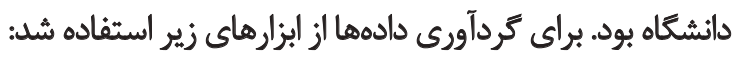

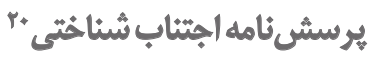

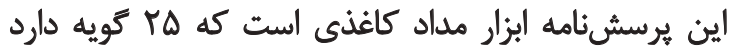

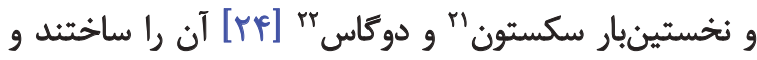

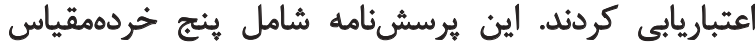

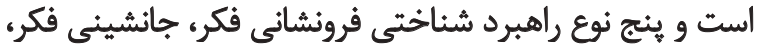

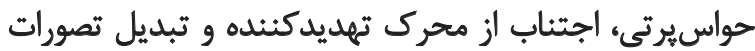

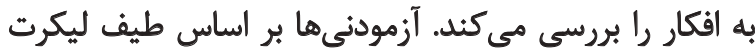

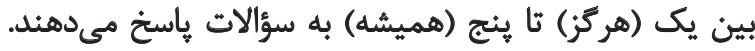

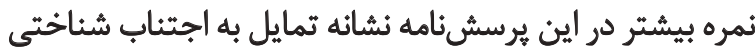

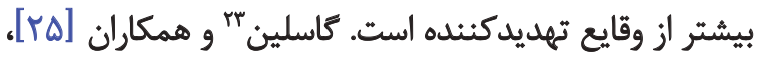

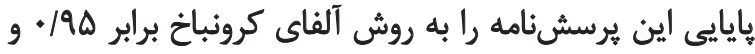

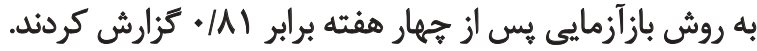

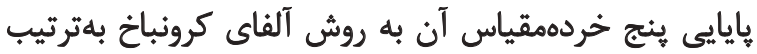

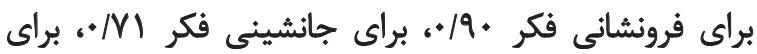

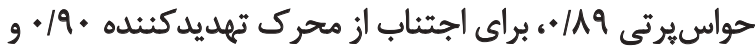

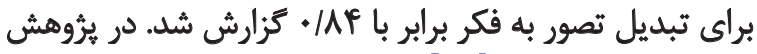

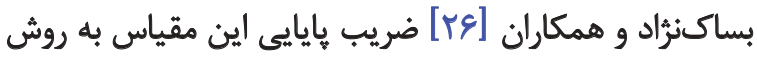

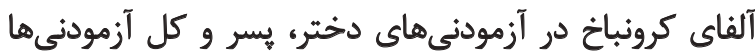

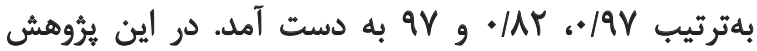

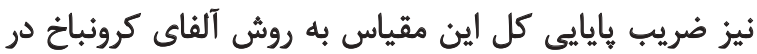

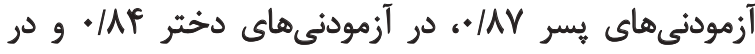

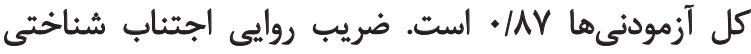

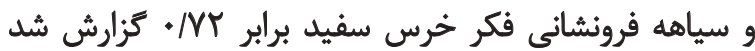

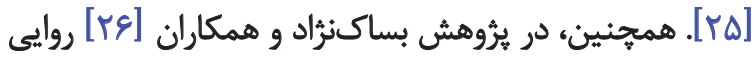

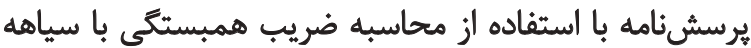

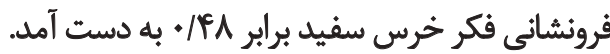

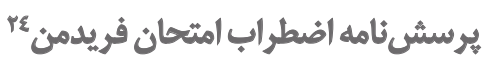

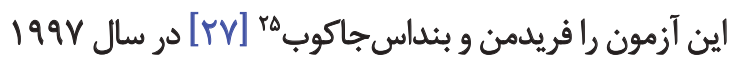

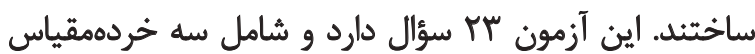

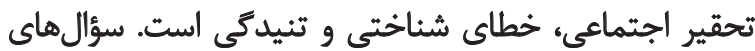

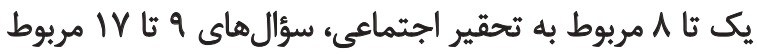

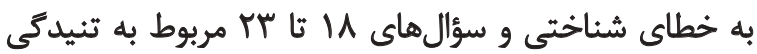

20. Cognitive Avoidance Questionnaire (CAQ)

21. Sexton

22. Dugas

23. Gosselin

24. Friedman Test Anxiety (FTA)

25. Fridman \& Bendas-Jacob

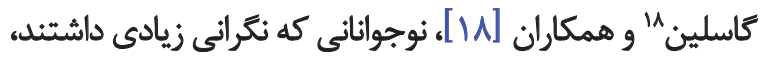

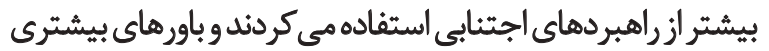

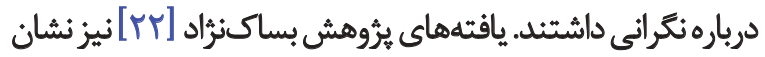

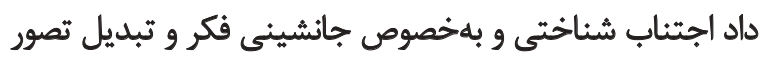

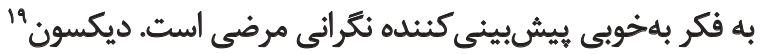

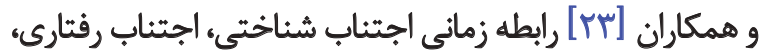

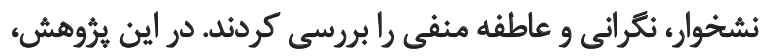

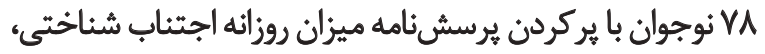

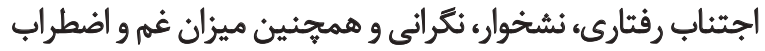

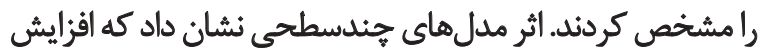

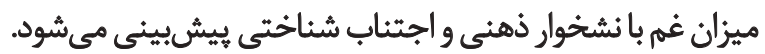

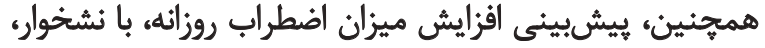

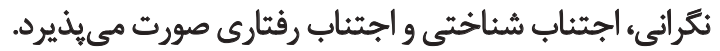
با توجه به تأثير اهمال كارى و اجتناب شناختي إنى در ايجاد

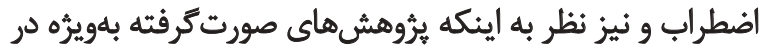

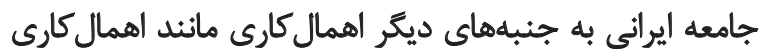

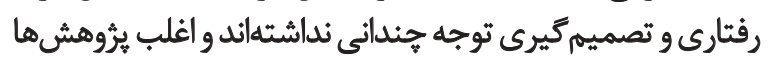

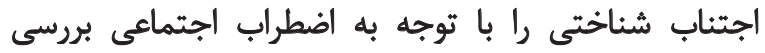

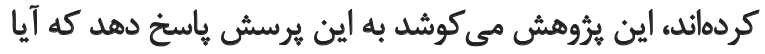

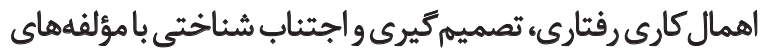

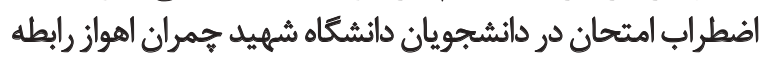

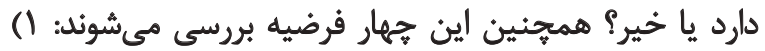

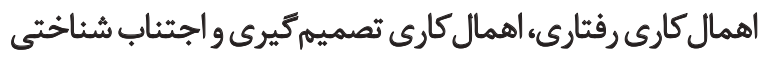

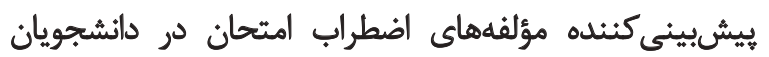

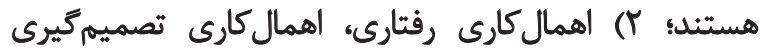

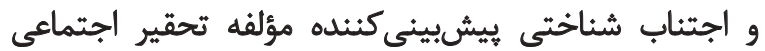

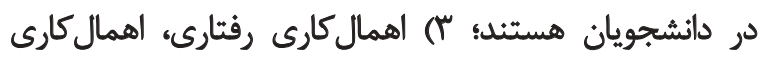

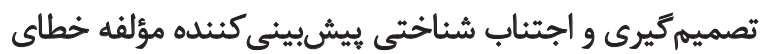

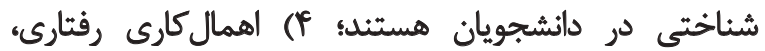

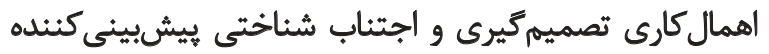
مؤلفه تنيدكى در دانشجويان هستيند. ووشُ

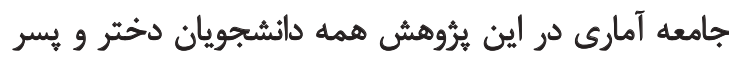

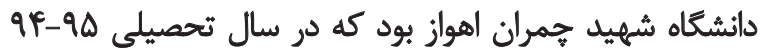

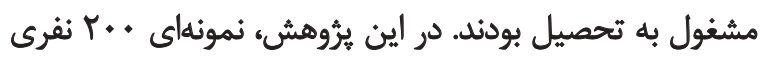

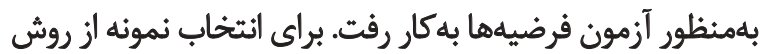

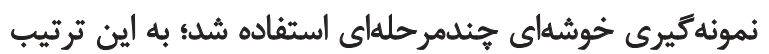

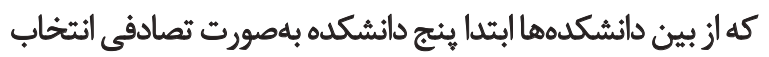

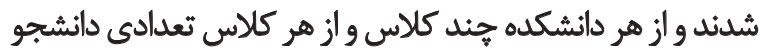

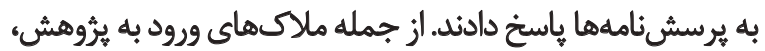

18. Gosseline

19. Dickson 


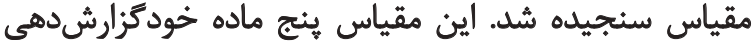

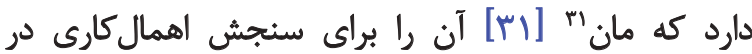

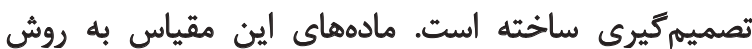

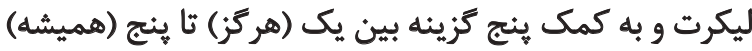

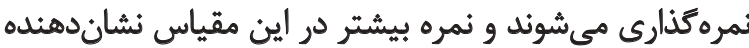

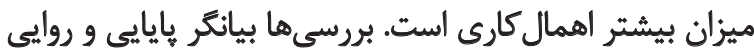

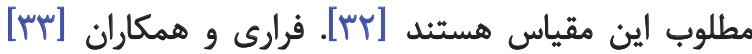

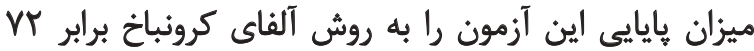

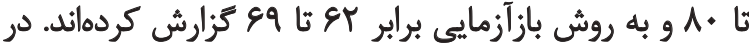

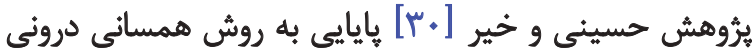

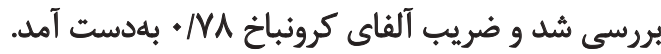

در اين يُروهش ميزان ثايايى اين مقياس به روش آلفاى كرونباخ

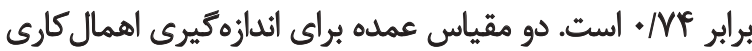

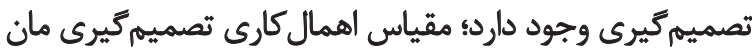

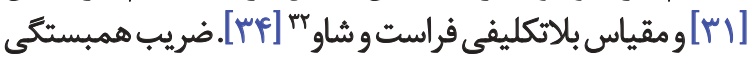

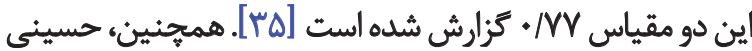

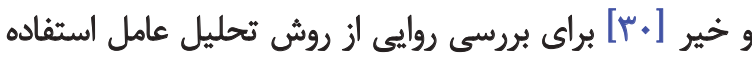

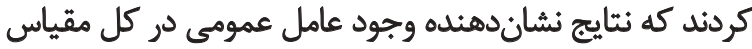

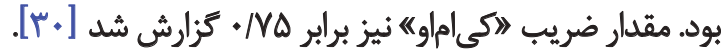

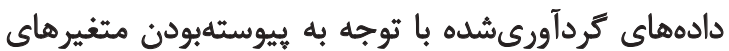

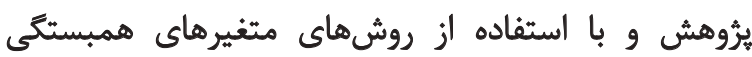

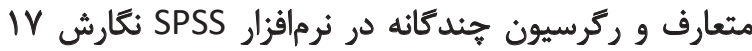

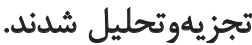

يافتههها

در جدول شماره ا شاخصهاى متغيرهاى بيشبين و ملاك

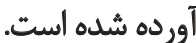

معنادارى مدل كامل تحليل همبستكي متعارف

نتايج آزمونهاى معنادارى مدل كامل تحليل همبستئى

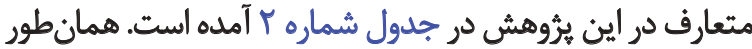

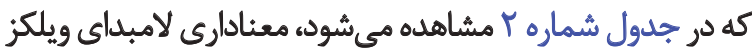

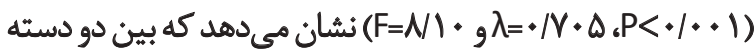

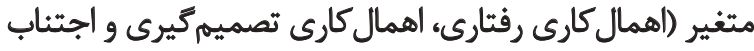

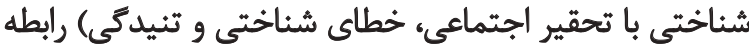

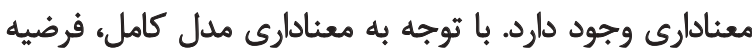

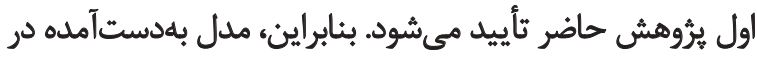

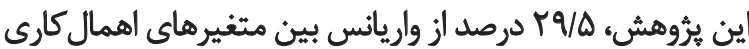

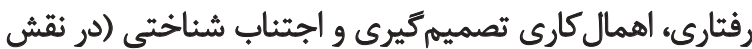
متغير بيشبين) با تحقير اجتماعى، خطاى شناختى و تنيدئى (دئي

31. Mann

32. Frost \& Shows
هستند. اين آزمون به روش ليكرت (از صفر تا سا) نمرهكذارى

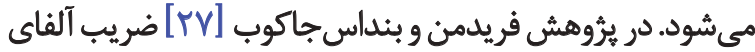

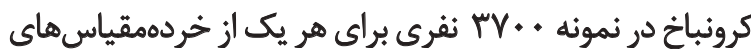

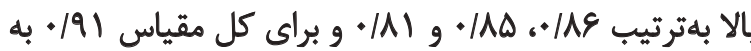

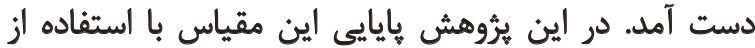

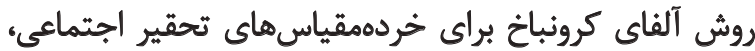

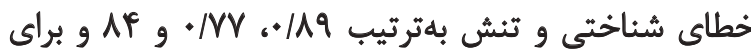

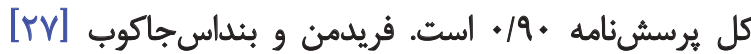

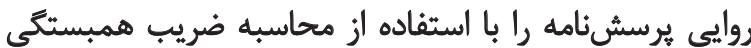

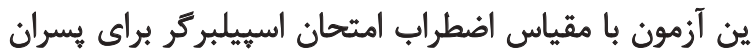

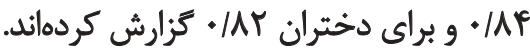

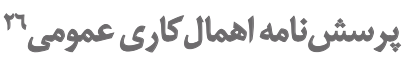

در اين يُروهش، براى سنجيدن اهمال كارى رفتارى، از

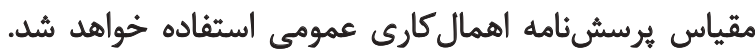

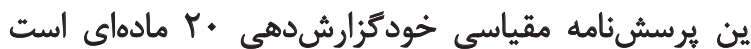

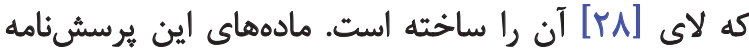

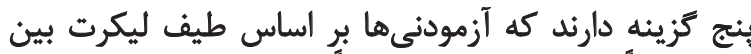

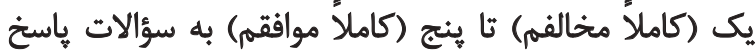

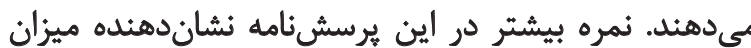

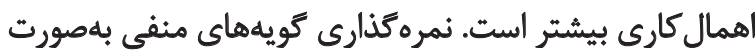

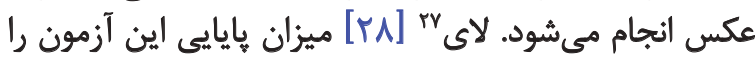

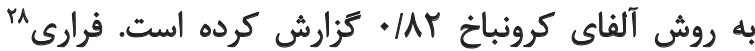

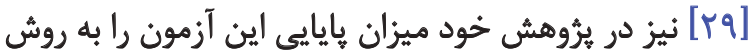

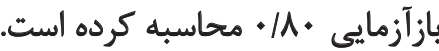

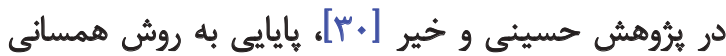

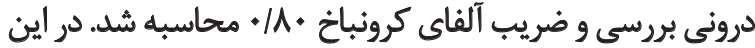

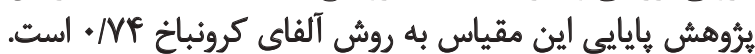

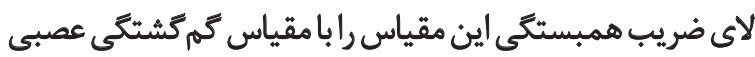

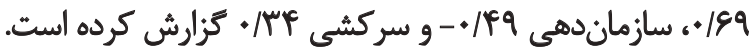

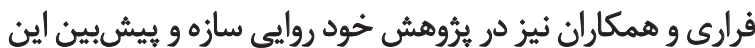

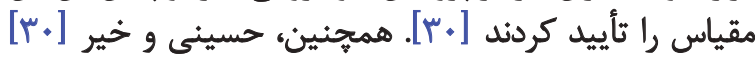
براى بررسى روايى از روش تحليل عامل استفاده كردئد كه نتايج،

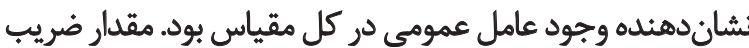

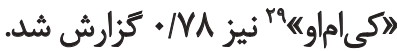

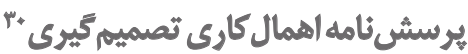

در اين يُروهش اهمال كارى در تصميمكيرى به كمك اين

26. General procrastination scale (GPS)

27. Lay

28. Ferrari

29. KMO

30. Decisional procrastination scale (DPS) 
جدول ا. ميانكين، ائحراف معيار، كمترين و بيشترين نمره متغيرها ( · (N=r)

\begin{tabular}{|c|c|c|c|c|c|}
\hline بيشترين نمره & كمثرين نمره & انحراف معيار & مياتكين & تعداد & مثغير \\
\hline va & rr & १/ह & $\Delta V / \& \Delta$ & $r+$. & اهمال كارى رثثارى \\
\hline ra & $\Delta$ & $r / q$. & $18 / 18$ & r... & اهمال كارى تصميمئيرى \\
\hline $11 \mathrm{~A}$ & ra & $1 / 19$ & VE/Ir & r.. & اجتثاب شناختمى \\
\hline re & - & $\Delta / M T$ & $11 / * f$ & r.. & تحقير اجتماعى \\
\hline M & - & P/19 & $1 . / m$ & r.. & خطاى شناختى \\
\hline M & - & $\varphi+8$ & e/er & $r+$. & تمنش \\
\hline$\Delta q$ & - & $1 / \cdot 9$ & rV/A. & $r+$. & اضطراب امتحان (كل) \\
\hline
\end{tabular}

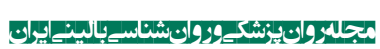

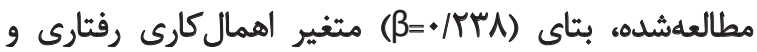

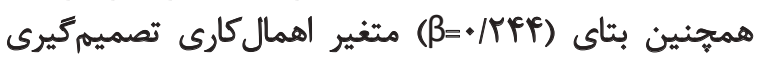

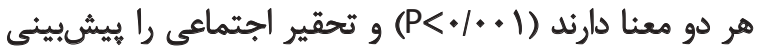

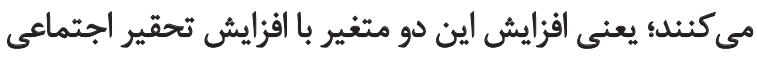

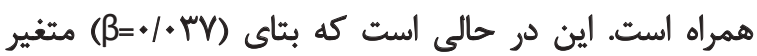

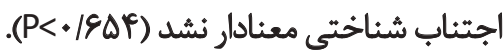

يافتههاى مريوط به آزمون فرضيه سوم: اهمال كارى رفتارى،

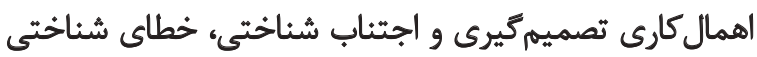

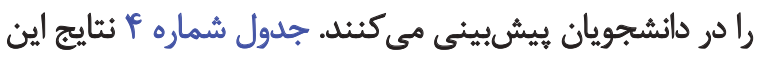
تحليل رانشان مي دهد.

همان Fونه كه در جدول شماره f أمشاهده مي شود، بر اساس

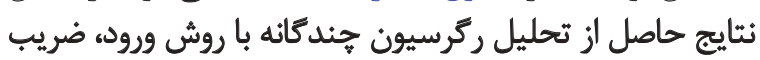

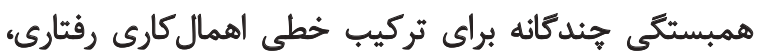

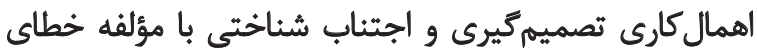

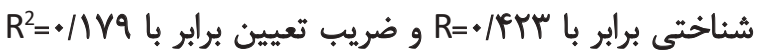

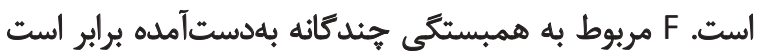

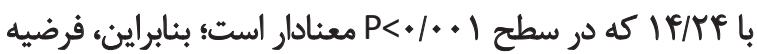

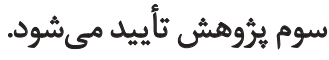

با توجه به ضريب تعيين بهدستآمده، حدود V/9 درصد

$$
\text { (در نقش متثير ملاكى) را تبيين مى كند. }
$$

بررسى فرضيههاي فرعى ثيروهش از طريق تحليل ركرسيون

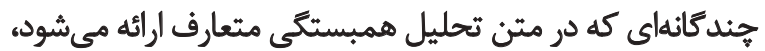

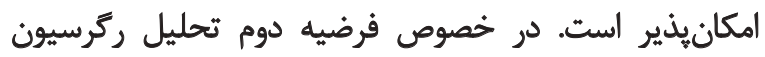

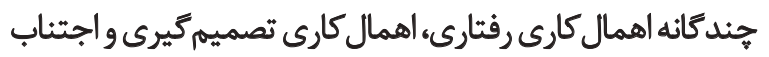

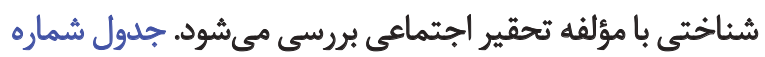

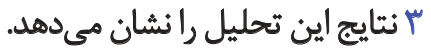

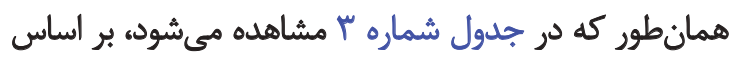
نتايج حاصل از تحليل ركرسيون زيند

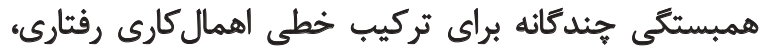

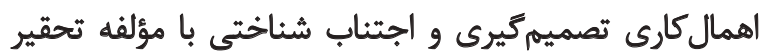

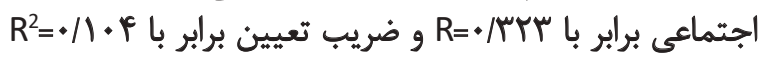

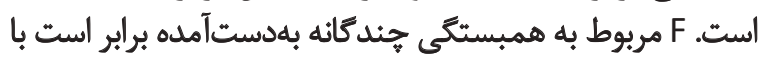

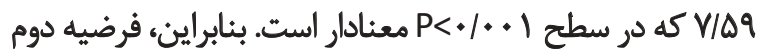

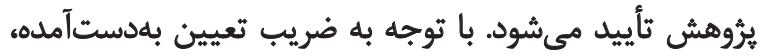

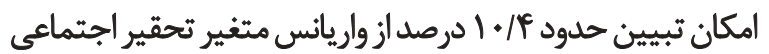

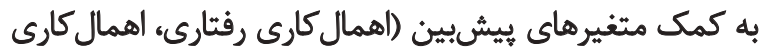
تصميم كيرى و اجتناب شناختي ) ميسر است

همان طور كه ملاحظه مىشود از ميان متغيرهاى بيشبين

جدول r. آزمونهاي معنادارى براي مدل كامل تحليل همبستكى متعارف

\begin{tabular}{|c|c|c|c|c|c|}
\hline سطح معنادارى & خطاdf & فرضيه df & $F$ & مقدار & نام آز مون \\
\hline .1 .01 & $\Delta M$ & 9 & V/NE &.$/ M M$ & اثر ييلايي \\
\hline $.1 . .1$ & PrT & 9 & N). & $\cdot / N \cdot \Delta$ & لامبداي ويلكز \\
\hline $.1 . .1$ & $\Delta \vee \Lambda$ & 9 & NMT & - ruar & اثر هتلينك \\
\hline- & - & - & - & $\cdot / r+1$ & ريشه روى (Roy) \\
\hline
\end{tabular}




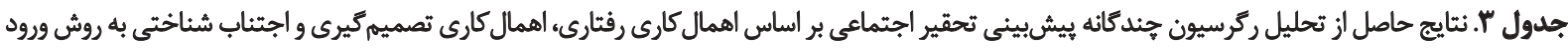

\begin{tabular}{|c|c|c|c|c|c|c|c|c|}
\hline $\mathbf{P}$ & $\mathbf{T}$ & $\beta$ & B & Pتمال P P & Fسبت F & $\mathbf{R}^{r}$ & $\mathbf{R}$ & متغيرهاى ييش آمارى \\
\hline .1 .01 & r/ea & . & . /ru & & & & & اهمال كارى رفتارى \\
\hline .1 .01 & Plea & $\cdot / r \cdot r$ &.$/ P I T$ & $P<+1+\cdot 1$ & $V / \Delta 9$ & $+11+4$ & 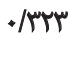 & اهمال كارى تصميم:كيرى \\
\hline .1940 &.$/ 1999$ & $.1 . \mathrm{W}$ & .1 .10 & & & & & اجتناب شناختي \\
\hline
\end{tabular}

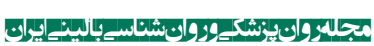

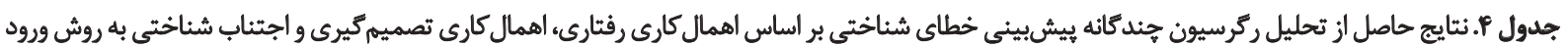

\begin{tabular}{|c|c|c|c|c|c|c|c|c|}
\hline $\mathbf{P}$ & $\mathbf{T}$ & $\beta$ & B & P Pالمال & Fسبت F & $\mathbf{R}^{r}$ & $\mathbf{R}$ & شاحضص آماري متغيرهاي \\
\hline .1 .01 & $\Delta / M q$ & $\cdot /$ PAl &.$/ 1 V^{e}$ & & & & & اهمال كارى رفثتارى \\
\hline .1 .08 & $r / v q$ & $\cdot M \cdot F$ &.$/ 419$ & $P<+\mid *+1$ & IF/MP &.$/ 1199$ & / & اهمال كارى تصميمكيرى \\
\hline $.1 \Delta 59$ & $-* / \cdot \Delta V$ &.$- /+40$ & $-.1 \cdot 1 f$ & & & & & اجتناب شناختى \\
\hline
\end{tabular}

مجلم:

نتايج حاصل از تحليل ركرسيون جند أنانه با روش ورود، ضريب

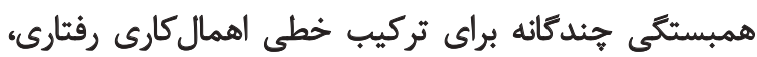

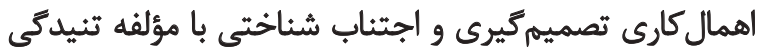

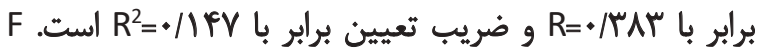

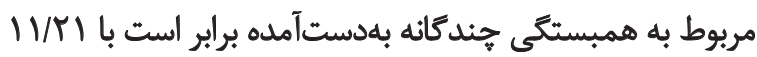

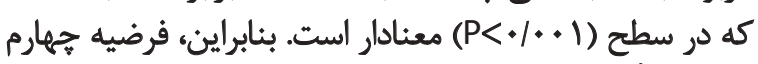

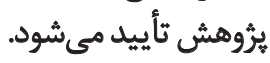

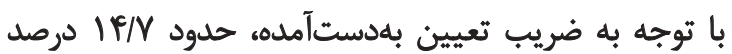

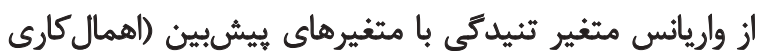

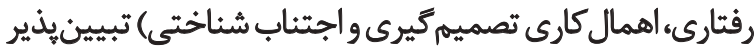

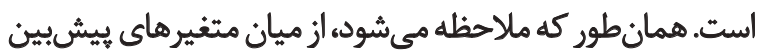

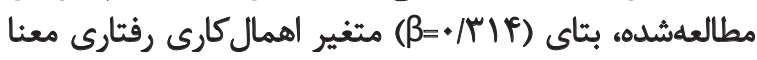

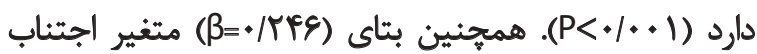

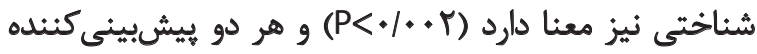

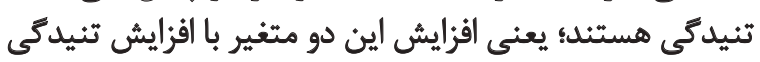

از واريانس متغير خطاى شناختى با متغيرهاي بيشبين إنبين

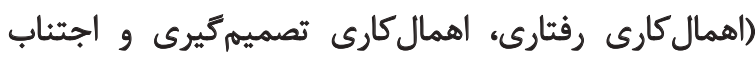

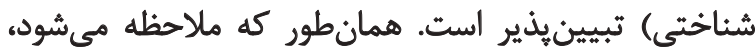

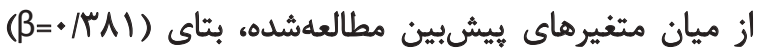

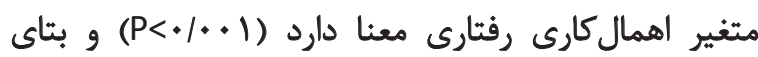

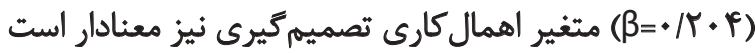

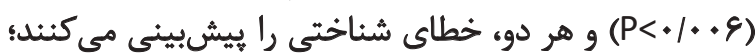

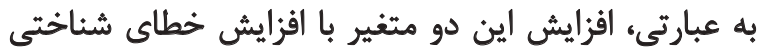

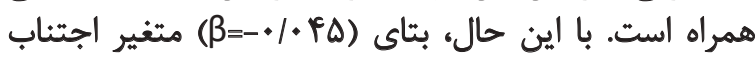

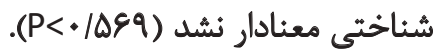
يافتههاى مربوط به آزمون فرضيه جهار م: اهمال كارى رفتّارى،

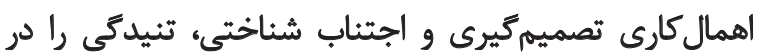

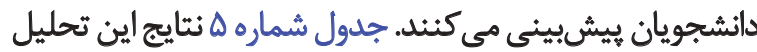
رانشان مي دهد. همانطور كه در جدول شماره ه مشاهده هي شود، بر اساس

جدول ه. نتايج حاصل از تحليل ركرسيون جيندكانه بيشبينى تنيدكى بر اساس اهمال كارى رفتارى، اهمال كارى تصميمكيرى و اجتناب شناختى بله روش ورود

\begin{tabular}{|c|c|c|c|c|c|c|c|c|}
\hline $\mathbf{P}$ & $T$ & $\beta$ & B & Pتمال P & نسبت F & $\mathbf{R}^{r}$ & $\mathbf{R}$ & متغيرهاي بيشي آمارى \\
\hline$+1+\infty$ & $f / f$ & .rutr &.$/ 14 q$ & & & & & اهمال كارى رفتارى \\
\hline . & I/Tr & /.95 & 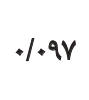 & $P<+1 .+1$ & $\mid W / r$ & - INP & שגז/. & تصميم: تهيرى \\
\hline $.10 . r$ & $r / \cdot \Lambda$ & . MPE & $.1 \cdot W$ & & & & & اجثناب شناختي \\
\hline
\end{tabular}




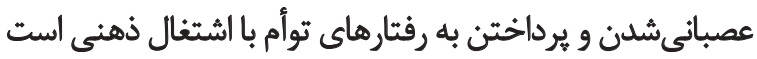

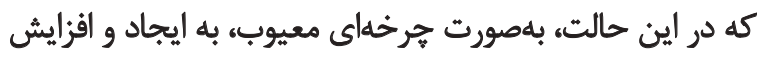
اضطراب امتحان در فرد منجر مىشود.

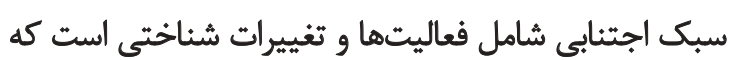

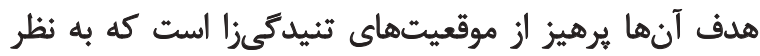

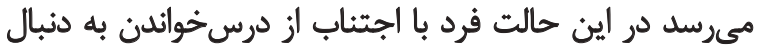

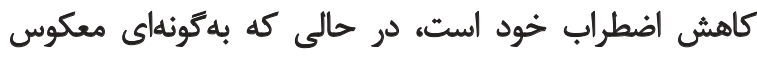

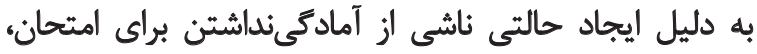

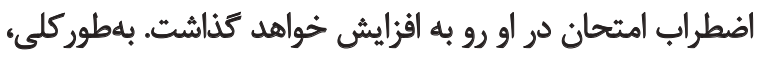

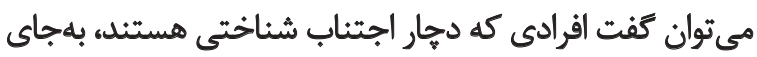

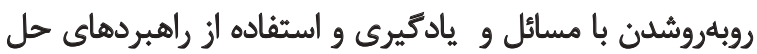

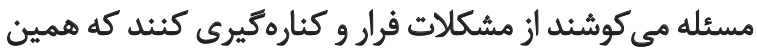

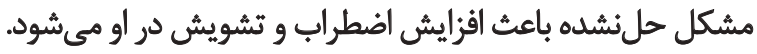

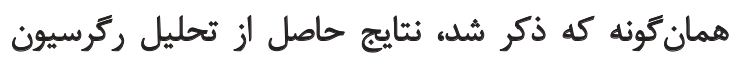

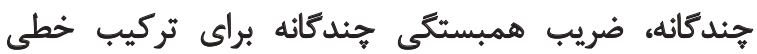

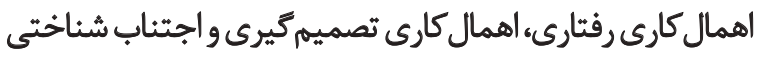

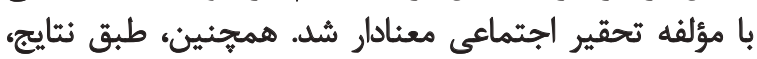

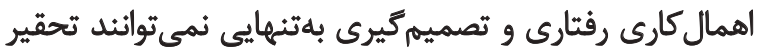

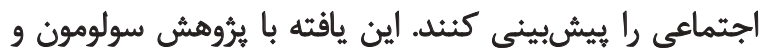

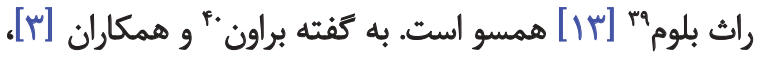

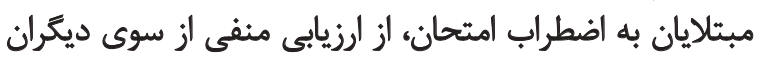

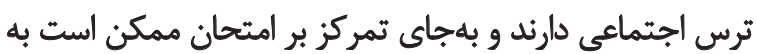

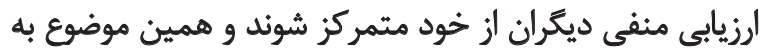

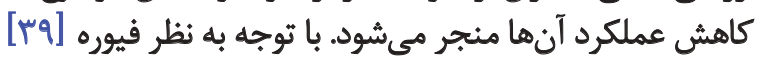

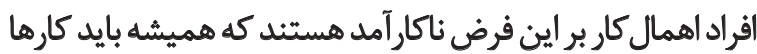

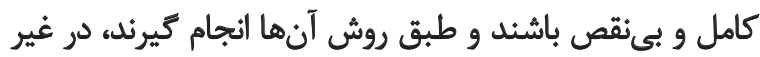

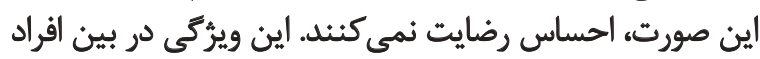

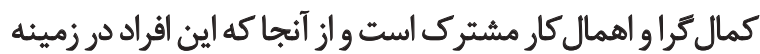

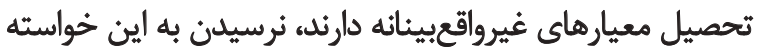

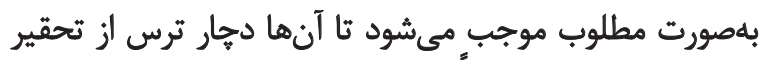

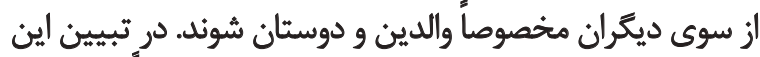

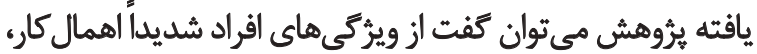

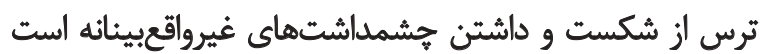

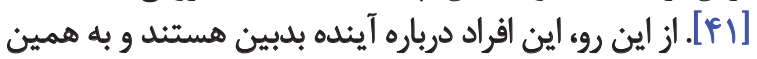

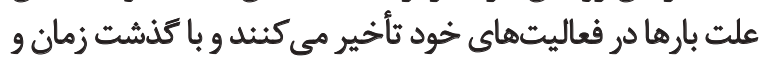

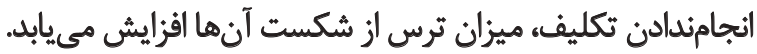

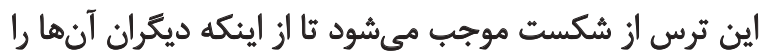

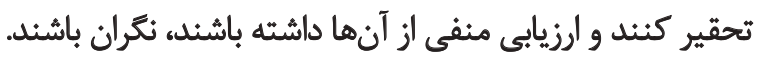

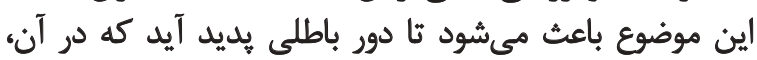

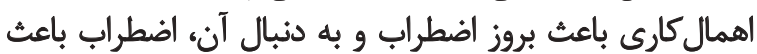

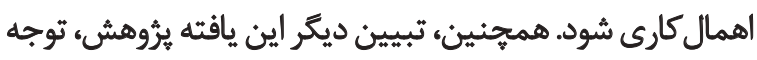

39. Solomon \& Rothblum 40. Brown

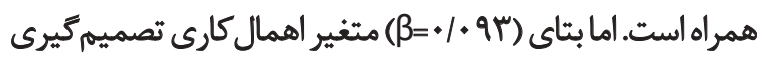

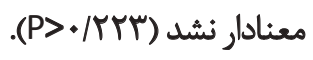

\&)

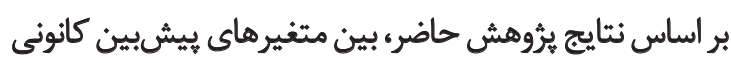

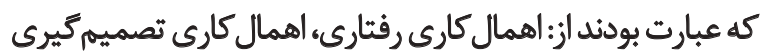

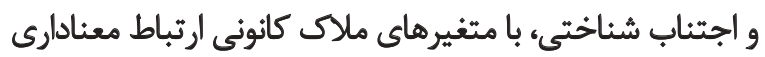

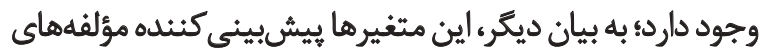

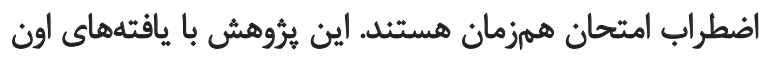

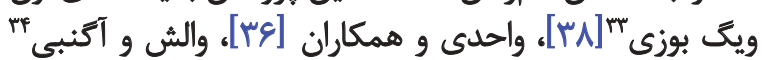

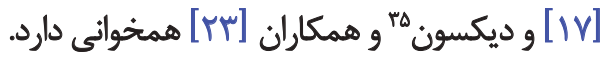

افراد اهمال كار مطالعه دروس را تا شب امتحان به تعويق

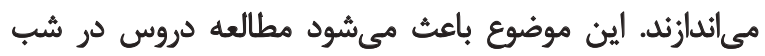

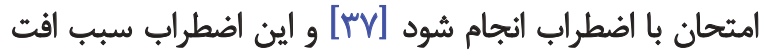

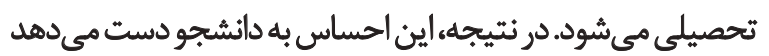

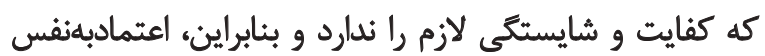
تحصيلي دانشُجو صلمهل جلى هي سئيند.

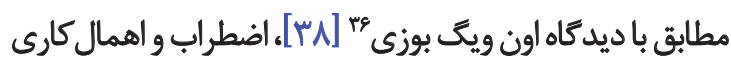

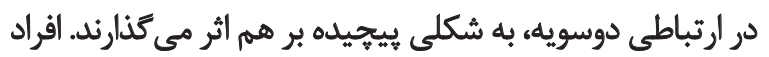

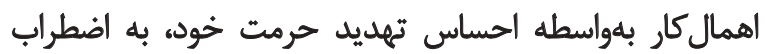

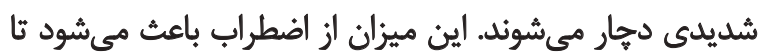

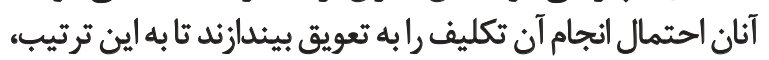

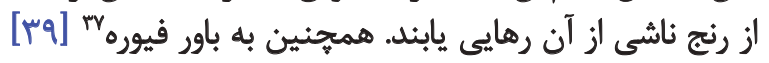

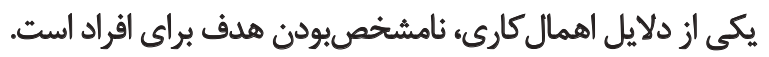

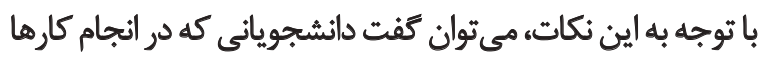

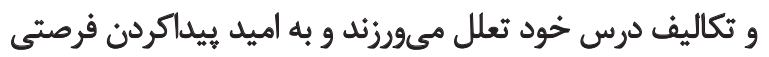

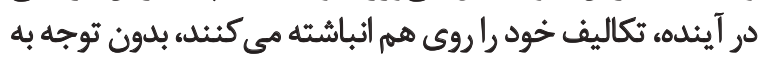

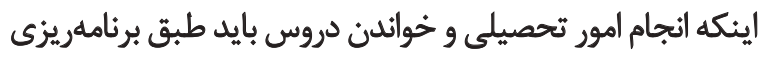

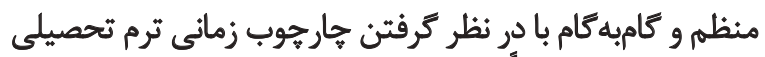

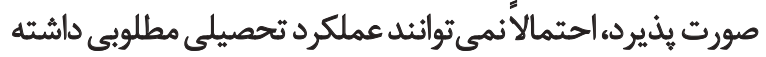

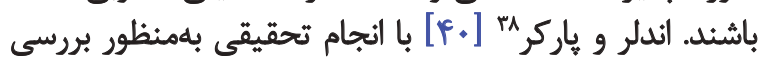

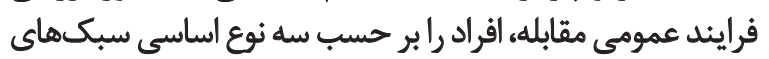

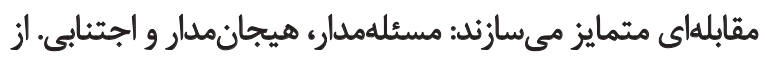

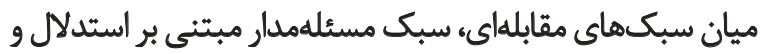
شامل جستوجوى اطلاعات درباره مسئله و تغيير ساختار مسار مسئله

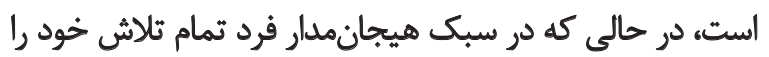

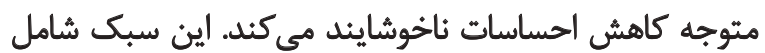

33. Onwuegbuzie

34. Walsh \& Agwunobi

35. Dickson

36. Onwuegbuzie

37. Fiore

38. Endler \& Parker 


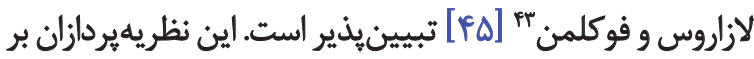

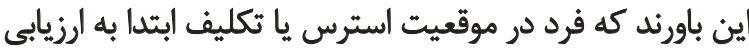

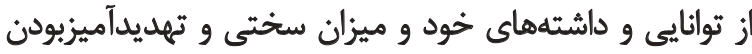

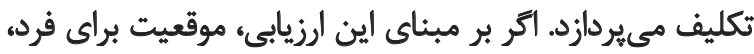

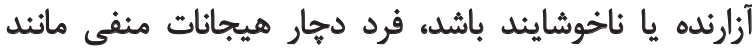

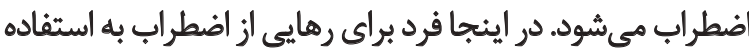

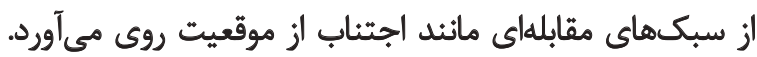

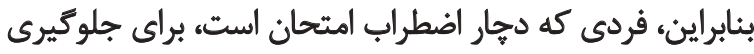

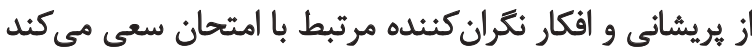

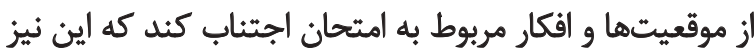

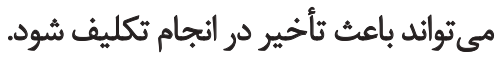

\section{نتيجلمَيرى}

به طور خلاصه، طبق يافتههاى ثبروهش حاضر، افراد ديار

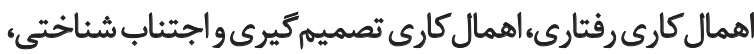

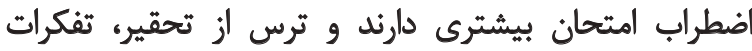

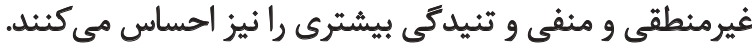

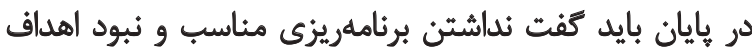

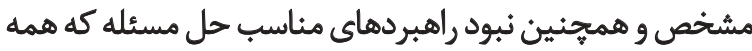

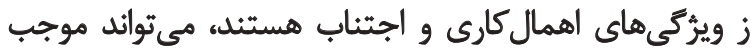

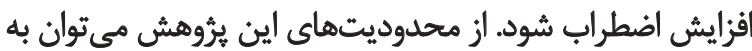

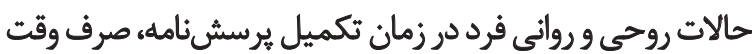

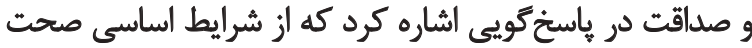

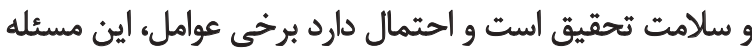

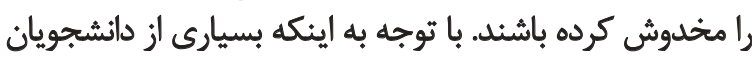

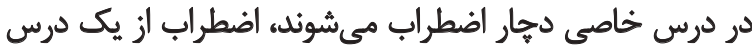

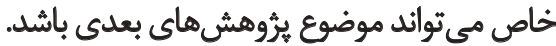

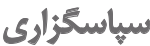

از استادان راهنما و مشاور و هميجنين همه دانشجويان

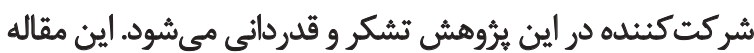

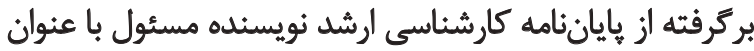

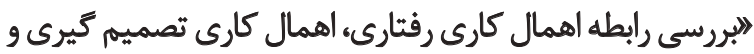

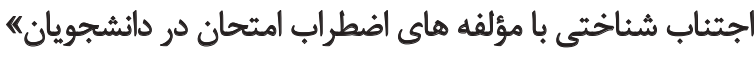

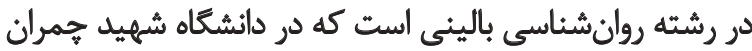

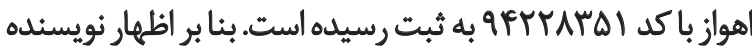

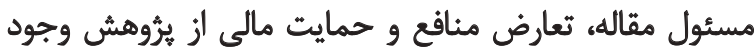

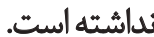

به اين نكته است كه يكى از ويرُكى هاى افراد شديداً اهمال كار، خودكارآمدى ضعيف است [FT]

همان كونه كه اشاره شد، نتايج حاصل از تحليل ركّرسيون

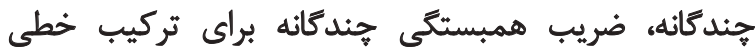

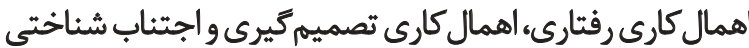

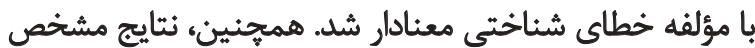

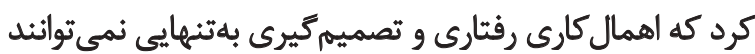

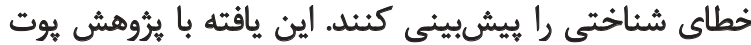

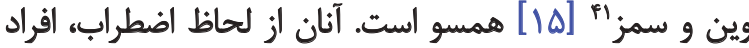

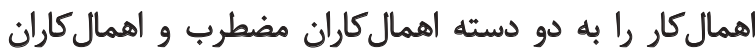

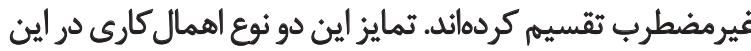

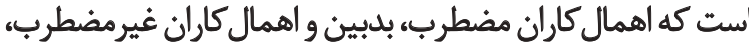

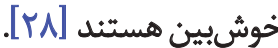

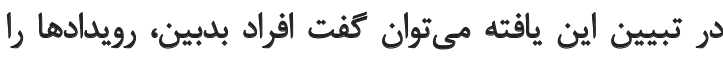

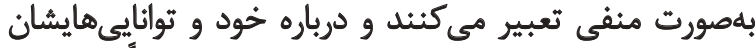

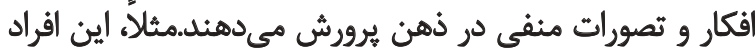

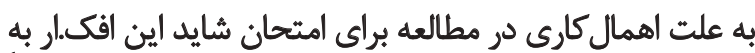

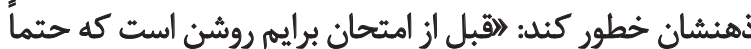

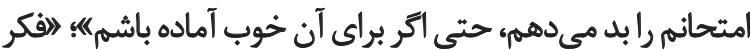

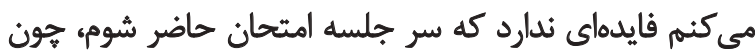

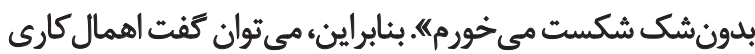

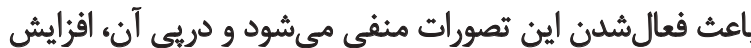

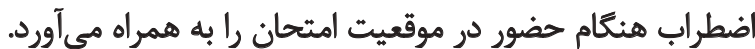

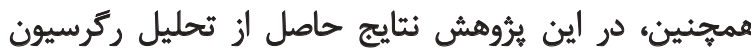

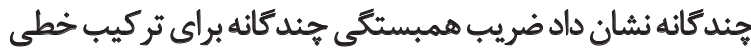

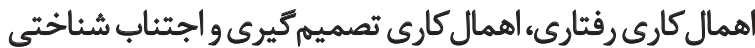

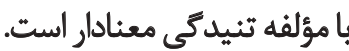

همجنين، بر اساس نتايج، اهمال كارى رفتارى و اجتناب

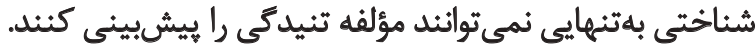

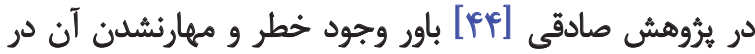

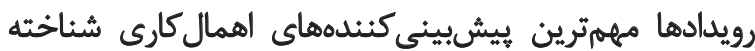

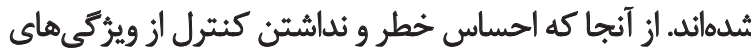

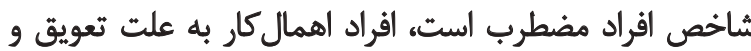

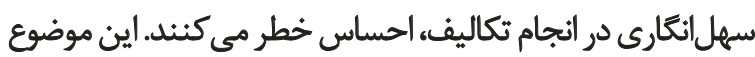

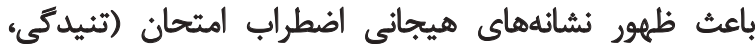

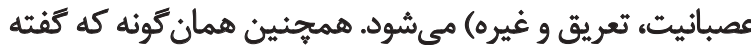

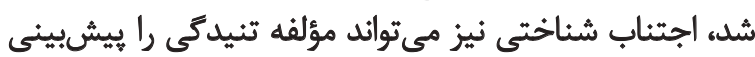

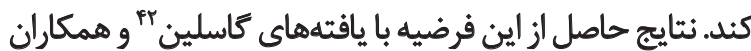

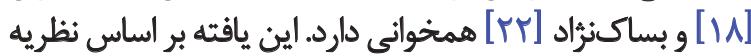

41. Putwain \& Symes

42. Gosseline 


\section{References}

[1] Abolghasemi A, Najarian B. [Test anxiety, assessment and treatment (Persian)]. Psychological Research. 2000; 5(3-4):82-99.

[2] Latas M, Pantic M, Bradovic D. Analysis of test anxiety in medical students. Medicinski Pregled. 2010; 63(11-12):863-6. doi: $10.2298 /$ mpns 10128631

[3] Brown LA, Forman EM, Herbert JD, Hoffman KL, Goetter EM. A randomized Controlled trial of acceptance-based behavior therapy and cognitive therapy for test anxiety: A pilot Study. Behavioural Modification. 2011; 35(1):31-53. doi: $10.1177 / 0145445510390930$

[4] Schaefer A, Matthess H, Pfitzer G, Kohle K. [Mental health and performance of medical students with high and low test anxiety (German)]. PPmP - Psychotherapie · Psychosomatik · Medizinische Psychologie. 2007; 57(7):289-97. doi: 10.1055/s-2006-951974

[5] Pless A. Treatment of test anxiety: A computerized approach [PhD dissertation]. Michigan: Centeral Michigan University; 2010.

[6] Morris LW, Liebert RM. Effects of anxiety on timed and untimed intelligence tests: Another look. Journal of Consulting and Clinical Psychology. 1969; 33(2):240-4. doi: 10.1037/h0027164

[7] Spilberger CD. Preliminary professional manual for the Test Anxiety Inventory. Palo Alto, California: Consulting Psychologist Press; 1980.

[8] Nasri S, Shahrokhi M, Ebrahim Damavandi M. [The prediction of academic procrastination on perfectionism and test anxiety (Persian)]. Research in School and Virtual Learning. 2013; 1(1):2637.

[9] Howell AJ, Watson DC. Procrastination: Associations with achievement goal orientation and learning strategies. Personality and Individual Differences. 2007; 43(1):167-78. doi: 10.1016/j. paid.2006.11.017

[10] Kim KR, Seo EH. The relationship between procrastination and academic performance: A meta-analysis. Personality and Individual Differences. 2015; 82:26-33. doi: 10.1016/j.paid.2015.02.038

[11] Ferrari JR, Ozer BU, Demir A. Chronic procrastination among Turkish adults: Exploring decisional, avoidant, and arousal styles. The Journal of Social Psychology. 2009; 149(3):302-08. doi: 10.3200 / socp. $149.3 .302-308$

[12] Sheykhi M, Fathabadi J, Heidari M. [The relations of anxiety, self-efficacy and perfectionism to dissertation procrastination (Persian)]. Developmental Psychology. 2013; 9(35):283-95.

[13] Solomon LJ, Rothblum ED. Academic procrastination: Frequency and cognitive behavioural correlates. Journal of Counselling Psychology. 1984; 31(4):503-9. doi: 10.1037//0022-0167.31.4.503

[14] Soysa CK, Weiss A. Mediating perceived parenting styles-test anxiety relationships: Academic procrastination and maladaptive perfectionism. Learning and Individual Differences. 2014; 34:7785. doi: $10.1016 /$ j.lindif.2014.05.004

[15] Putwain D, Symes W. Perceived fear appeals and examination performance: Facilitating or debilitating outcomes? Learning and Individual Differences. 2011; 21(2):227-32. doi: 10.1016/j.lindif.2010.11.022

[16] Ferrari JR, Dovidio JF. Examining behavioral processes in indecision: Decisional Procrastination and decision- making style. Journal of Research in Personality. 2000; 34(1):127-37. doi: 10.1006/jrpe.1999.2247

[17] Walsh JJ, Ugumba-Agwunobi G. Individual differences in statistics anxiety: The roles of perfectionism, procrastination and trait anxiety. Personality and Individual Differences. 2002; 33(2):239-51. doi: 10.1016/s0191-8869(01)00148-9

[18] Gosselin P, Langlois F, Freeston MH, Ladouceur R, Laberge M, Lemay D. Cognitive variables related to worry among adolescents: Avoidance strategies and faulty beliefs about worry. Behaviour Research and Therapy. 2007; 45(2):225-33. doi: 10.1016/j. brat.2006.03.001

[19] Bassak-nejad S, Hooman F, Ghasemi-nejad MA. [The relationship between cognitive-behavioral avoidance coping styles with eating disorder among university students (Persian)]. Journal of Fundamentals of Mental Health. 2013; 14(56):278-85.

[20] Beesdo-Baum K, Jenjahn E, Höfler M, Lueken U, Becker ES, Hoyer J. Avoidance, safety behavior, and reassurance seeking in generalized anxiety disorder. Depression and Anxiety. 2012; 29(11):948-57. doi: 10.1002/ da.21955

[21] Moini N. [A study of relationship between post event processing and cognitive avoidance with social anxiety among students of Shahid Chamran university of Ahwaz (Persian)] [MSc. thesis]. Ahwaz: Shahid Chamran University of Ahwaz; 2010.

[22] Bassak Nejad S. The relationship between cognitive avoidance and pathological worry among university students. Paper presente at: $23^{\text {rd }}$ Conference of EHPS. 15-19 September 2009; Pisa, Italy.

[23] Dickson KS, Ciesla JA. Reilly LC. Rumination, worry, cognitive avoidance, and behavioral avoidance: Examination of temporal effects. Behavior Therapy. 2012; 43(3):629-40. doi: 10.1016/j. beth.2011.11.002

[24] Sexton, KA. Dugas MJ. The cognitive avoidance questionare: Vlidetion of the English translation. Journal of Anxiety Disorder. 2008; 22(3):355-70. doi: 10.1016/j.janxdis.2007.04.005

[25] Gosselin P, Langlois F, Freeston MH, Ladouceur R, Dugas MJ, Pelletier O. [The Cognitive Avoidance Questionnaire(CAQ): Development and validation among adult and adolescent samples (French)]. Journal de The'rapie Comportementale et Cognitive 2002; 12(1):24-37.

[26] Bassak-nejad S, Moini N, Mehrabizadeh-Honarmand M. [The relationship between post event processing and cognitive avoidance with social anxiety among students (Persian)]. International Journal of Behavioral Sciences. 2011; 4(4): 335-40.

[27] Fridman I, Bendas-Jacob O. Measuring precieved test anxiety inadolescents: A self-report scale. Educational and Psychological Measurement. 1997; 57(6):1035-46. doi: $10.1177 / 0013164497057006012$

[28] Lay CH. At last, my research article on procrastination. Journal of Research in Personality. 1986; 20(4):474-95. doi: 10.1016/00926566(86)90127-3

[29] Ferrari JR. Reliability of academic and dispositional measure of procrastination. Psychological Reports. 1989; 64(3_suppl):1057-8. doi: $10.2466 /$ pr0.1989.64.3c.1057

[30] Hosseine F, Khayyer M. [Prediction of behavioral and decisional procrastination considering meta-cognition beliefs in university students (Persian)]. Iranian Journal of Psychiatry \& Clinical Psychology. 2009; 15(3):265-73. 
[31] Mann L. Decision-making questionnaire. Unpublished inventory. Adelaide: Flinders University; 1982.

[32] Spada MM, Hiou K, Nikcevic AV. Meta cognitions, emotions, and procrastination. Journal of Cognitive Psychotherapy. 2006; 20(3):319-26. doi: 10.1891/jcop.20.3.319

[33] McCown WG, Ferrari JR, Johnson J. Procrastination and task avoidance: Theory, research, and treatment. Berlin: Springer; 1995.

[34] Frost RO, Shows DL. The nature and measurement of compulsive indecisiveness. Behaviour Research and Therapy. 1993; 31(7):683-92. doi: 10.1016/0005-7967(93)90121-a

[35] Zeisler L. Association between stress and decisional procrastination in parents of children with down syndrome during their developemental transitions [PhD dissertations]. South Orange, New Jersey: Seton Hall University; 2011.

[36] Vahedi SH, Farrokhi F, Gahramani F, Issazadegan A. The relationship between procrastination, Learning strategies and statistics anxiety among Iranian college student: A canonical correlation analysis. Iranian Journal of Psychiatry and Behavioral Sciences. 2012; 6(1):40-46

[37] Rothblum ED, Solomon LJ, Murakami J. Affective, cognitive, and behavioral differences between high and low procrastinators. Journal of Counseling Psychology. 1986; 33(4):387-94. doi: 10.1037/0022-0167.33.4.387

[38] Onwuegbuzie AJ. Academic procrastination and statistics anxiety. Assessment and Evaluation in Higher Education. 2004; 29(1):3-19. doi: 10.1080/0260293042000160384

[39] Fiore N. The now habit: A strategic program for overcoming procrastination and enjoying guilt-free play. New York: Penguin Group; 1989.

[40] Endler NS, Parker JD. Multidimensional assessment of coping: A critical evaluation. Journal of Personality and Social Psychology. 1990; 58(5):844-54. doi: 10.1037/0022-3514.58.5.844

[41] Balkis M, Duru E. The evaluation of the major characteristics and aspects of the procrastination in the framework of psychological counseling and guidance. Educational Sciences: Theory \& Practice 2007; 7(1):376-85.

[42] Chu AC, Chol JN. Rethinking procrastination: Positive effects of active procrastination behavior on attiudes and performance. Journal of Social Psychology. 2005; 145(3):245-64. doi: 10.3200/ socp.145.3.245-264

[43] Lay CH. The relationship of procrastination and optimism to judgments of time to complete an essay and anticipation of setbacks. Journal of Social Behavior \& Personality. 1988; 3(3):201-14.

[44] Sadeghi $\mathrm{H}$. The study of relation between meta cognition beleifs and procrastination among students of Tabriz and Mohaghegh Ardabili Universities. Procedia - social and Behavioral Sciences. 2011; 30:287-91. doi: 10.1016/j.sbspro.2011.10.057

[45] Lazarus RS, Folkman S. Stress, appraisal, and coping. Berlin: Springer; 1984 
